\title{
Membranschalen gleicher Festigkeit
}

\section{Doctoral Thesis}

Author(s):

Issler, Werner

Publication date:

1964

Permanent link:

https://doi.org/10.3929/ethz-a-000088859

Rights / license:

In Copyright - Non-Commercial Use Permitted 


\title{
MEMBRANSCHALEN GLEICHER FESTIGKEIT
}

\author{
VON DER \\ EIDGENÖSSISCHEN TECHNISCHEN \\ HOCHSCHULE IN ZÜRICH \\ ZUR ERLANGUNG \\ DER WÜRDE EINES DOKTORS \\ DER TECHNISCHEN WISSENSCHAFTEN
}

GENEHMIGTE

\section{PROMOTIONSARBEIT}

\author{
VORGELEGT VON \\ WERNER ISSLER \\ DIPL. -MATH. E. T. H. \\ VON DAVOS (KT. GRAUBÜNDEN)
}

REFERENT: HERR PROF. DR. H. ZIEGLER

KORREFERENT: HERR PROF. DR. B. THÜRLIMANN

SPRINGER-VERLAG - BERLIN/GÖTTINGEN/HEIDELBERG/NEW YORK 
1. Der Membranspannungszustand einer Rotationsschale. Wir betrachten eine stetig gekrümmte Fläche mit ihren Flächennormalen. Trägt man auf jeder Normale eine kleine Strecke $t$ ab, die durch den Durchstoßpunkt der Normale mit der Fläche halbiert wird und deren Länge stetig von den Flächenparametern abhängt, so begrenzen die Endpunkte der Strecken einen Körper, den man als Schale bezeichnet. Die Ausgangsfläche ist die Mittelfläche und $t$ die Schalenstärke der Schale.

Speziell versteht man unter einer Rotationsschale - auf solche wollen wir uns fortan beschränken - eine Schale, deren Mittelfläche eine Rotationsfläche ist und deren Schalenstärke $t$ längs den Breitenkreisen der Mittelfläche konstant bleibt (Abb. 1).

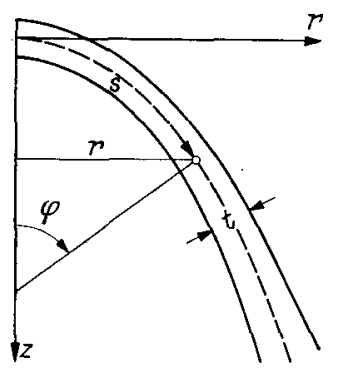

Abb. 1. Meridianschnitt einer Rotationsschale.

Mit Hilfe der cartesischen Koordinaten $x, y, z$ und der dazugehörigen Zylinderkoordinaten $r, \vartheta, z$ stellt man eine Rotationsfläche gewöhnlich in der Form

$$
\begin{aligned}
& x=r(s) \cos \vartheta \\
& y=r(s) \sin \vartheta, \\
& z=z(s)
\end{aligned}
$$

dar, wobei man mit $s$ die Bogenlänge auf dem Meridian bezeichnet.

Um die Membrantheorie formulieren und die folgenden Abschnitte in einfacher Form darstellen zu können, wollen wir die Zahl der möglichen Rotationsflächen durch drei Annahmen beschränken. Wir setzen voraus, daß

1. die Funktionen $r=r(s)$ und $z=z(s)$ mindestens zweimal stetig nach $s$ differentiierbar sind,

2. die Gausssche Krümmung der Fläche überall positiv ist und

3. die Rotationsfläche mit der Rotationsachse genau einen Punkt gemeinsam hat.

Die erste Annahme besagt einfach, daß die Fläche überall stetig gekrümmt ist. Ohne diese Voraussetzung können unstetige S,chnittkräfte auftreten. Für diesen Fall liefert die Membrantheorie ungenügende Resultate ${ }^{1}$. Auch unsere zweite Voraussetzung ist keine schwerwiegende Einschränkung, da die Membrantheorie für Schalen mit negativer Gaussscher Krümmung oft keine eindeutigen Lösungen liefert ${ }^{2}$. Das dritte Postulat verlangt, daß die Rotationsfläche einfach zusammenhängend ist. Kuppeln, die oben eine Öffnung besitzen, werden damit ausgeschlossen. Die wesentlichen Überlegungen der folgenden Abschnitte lassen sich auch auf solche Schalen übertragen. Wir verzichten aber auf die Behandlung dieses Problemkreises, um gewisse Untersuchungen der Abschnitte 3 und 5 in einfacher Form durchführen zu können.

Die Kurven $\vartheta=$ const und $s=$ const sind die Hauptkrümmungslinien der Fläche. Da die Gausssche Krümmung das Produkt der beiden Hauptkrümmungen ist, haben diese nach der zweiten Annahme des gleiche Vorzeichen. Durch eine geeignete Orientierung der Koordinaten (Abb. 1) kann man erreichen, daß die Hauptkrümmungen positiv ausfallen. Für die Krümmung des Meridians gilt dann die Beziehung

$$
k=\frac{d \varphi}{d s}>0
$$

für alle $s$. Dank dieser Ungleichung ist die Gleichung

$$
\varphi(s)=\arccos \frac{d r(s)}{d s}
$$

nach $s$ auflösbar, und daher kann der Winkel $\varphi($ Abb. 1) als Parameter eingeführt werden, wie es in der Schalentheorie üblich ist. Weil wir die Funktion $r=r(s)$ als zweimal stetig differentiierbar

1 W. Flügge, Statik und Dynamik der Schalen, 2. Aufl., S. 28, Berlin, Göttingen, Heidelberg: Springer 1957.

2 W. S. Wlassow, Allgemeine Schalentheorie und ihre Anwendung in der Technik, S. 22, Berlin 1958. 
vorausgesetzt haben, ist die neue Funktion $r=r(\varphi)$ - wie zum Beispiel aus (1.2) ersichtlich mindestens einmal stetig nach $\varphi$ differentiierbar.

Die Form des Meridians ist durch die Funktion

$$
r=r(\varphi)
$$

und die geometrische Bedeutung von $\varphi$ eindeutig bestimmt. Unter Beachtung der Definitionsgleichung

$$
\frac{d z}{d r}=\operatorname{tg} \varphi
$$

erhält man die Funktion $z=z(\varphi)$ durch die Integration

$$
z=\int_{0}^{\varphi} \operatorname{tg} \psi \frac{d r}{d \psi} d \psi .
$$

Die Hauptkrümmungsradien $r_{1}$ (des Meridians) und $r_{2}$ erhält man, durch den Parameter $\varphi$ ausgedrückt (Äb. 2), zu

$$
r_{1}=\frac{1}{\cos \varphi} \frac{d r}{d \varphi}>0
$$

und

$$
r_{2}=\frac{r}{\sin \varphi}>0
$$

Die Ungleichheitszeichen gelten wegen der zweiten Voraussetzung und der Wahl des Vorzeichens von (1.1). Aus (1.7) und der Voraussetzung 3 folgt, daß für den Parameter $\varphi$ höchstens das Intervall

in Frage kommt.

$$
0 \leq \varphi<\pi
$$

Den Durchstoßpunkt der Rotationsachse mit der Rotationsfläche bezeichnet man als den Scheitel der Schale. In diesem Punkt $(\varphi=0)$ stimmen die beiden Hauptkrümmungsradien überein, $\mathrm{d}$. h. es gilt die Beziehung

$$
r_{1}(0)=r_{2}(0)>0 \text {. }
$$

Wir betrachten nun ein Schalenelement, das durch zwei benachbarte Meridiane und Breitenkreise herausgeschnitten wird (Abb. 2). Die Größen $N_{\varphi}, N_{\vartheta}, N_{\varphi \vartheta}$ sind die Schnittkräfte pro Längeneinheit und $p_{\varphi}, p_{r}, p_{\vartheta}$ die Lasten pro Flächeneinheit. Die Membrantheorie nimmt an, daß die Spannungen an den Schnittflächen auf die obigen Schnittkräfte reduziert werden können. Das ist möglich, wenn der Spannungszustand eben und längs einer Mittelflächennormale konstant ist.

Die Gleichgewichtsbedingungen für das Schalenelement liefern die nötigen Gleichungen für die Schnittkräfte. Man erhält für die Richtung der Meridiantangente die partielle Differentialgleichung

$$
\frac{\partial}{\partial \varphi}\left(r \cdot N_{\varphi}\right)+r_{1} \frac{\partial N_{\varphi \vartheta}}{\partial \vartheta}-r_{1} N_{\vartheta} \cos \varphi+p_{\varphi} r r_{1}=0
$$

und entsprechend für die Richtungen der Breitenkreistangente und Mittelflächennormale

$$
\begin{aligned}
\frac{\partial}{\partial \varphi}\left(r \cdot N_{\varphi \vartheta}\right)+r_{1} \frac{\partial N_{\vartheta}}{\partial \vartheta}+r_{1} N_{\varphi \vartheta} \cos \varphi+p_{\vartheta} r r_{1} & =0, \\
\frac{N_{\varphi}}{r_{1}}+\frac{N_{\vartheta}}{r_{2}} & =p_{r} \cdot \mathbf{1}
\end{aligned}
$$

Wir setzen weiter voraus, daß die Schale rotationssymmetrisch belastet und gelagert und $p_{\vartheta}$ gleich null ist. Der ersten Voraussetzung zufolge verschwinden alle Ableitungen nach $\vartheta$, und (1.11) reduziert sich auf die Beziehung

$$
\frac{\partial}{\partial \varphi}\left(r N_{\varphi \vartheta}\right)+r_{1} N_{\varphi \vartheta} \cos \varphi=0,
$$

die durch $N_{\varphi \theta}=0$ befriedigt wird. Denkt man sich die Schale längs eines Breitenkreises aufgeschnitten und die Schnittkräfte angebracht, so folgt aus der Momentenbedingung bezüglich der Rotationsachse sofort die Eindeutigkeit der obigen Lösung.

1 W. Flügge, Statik und Dynamik der Schalen, 2. Aufl., S. 24, Berlin, Göttingen, Heidelberg: Springer 1957. 
Die Differentialgleichungen (1.10) und (1.12) vereinfachen sich damit zu

$$
\frac{d}{d \varphi}\left(r N_{\varphi}\right)-r_{1} \cos \varphi N_{\vartheta}=-p_{\varphi} r r_{1}
$$

und

$$
\frac{N_{\varphi}}{r_{1}}+\frac{N_{\vartheta}}{r_{2}}=p_{r}
$$

Eliminiert man aus (1.13) und (1.14) die Schnittkraft $N_{\theta}$, und beachtet man noch (1.7), so erhält man die wichtige Integraldarstellung für die Schnittkraft

$$
N_{\varphi}=\frac{1}{r_{2} \sin ^{2} \varphi}\left[\int r_{1} r_{2}\left(p_{r} \cos \varphi \cdots p_{\varphi} \sin \varphi\right) \sin \varphi d \varphi+c\right] \text {. }
$$

Die Integrationskonstante $c$ bestimmt sich aus der Forderung, daß die Schnittkräfte im Scheitel endlich bleiben müssen.

Sobald man die Schnittkräfte kennt, findet man die Spannungen mit den Beziehungen

$$
\sigma_{\varphi}=\frac{N_{\varphi}}{t}, \quad \sigma_{\vartheta}=\frac{N_{\vartheta}}{t} .
$$

Die Spannungen $\sigma_{\varphi}$ und $\sigma_{\theta}$ sind Hauptspannungen.

2. Rotationsschalen gleicher Festigkeit. Man spricht üblicherweise ${ }^{1}$ von einer Schale gleicher Festigkeit, wenn für jeden Punkt der Schale die Gleichung

$$
\sigma_{\varphi}=\sigma_{\vartheta}= \pm \sigma_{0}
$$

erfüllt ist, wobei mit $\sigma_{0}$ die Fließgrenze (bzw. Bruchgrenze) bei reinem Zug bzw. Druck bezeichnet ist.

Die Gleichungen (1.13), (1.14), (1.16) und die Bedingung der gleichen Festigkeit (2.1) legen für eine gegebene Belastung $p_{r}(\varphi), p_{p}(\varphi)$ die Meridianform $r=r(\varphi)$ der Mittelfläche und die Schalenstärke $t=t(\varphi)$ vollständig fest.

Besteht für eine drehsymmetrische Schale die Belastung nur aus dem Eigengewicht $(\gamma=$ spezifisches Gewicht des Materials durchwegs als konstant vorausgesetzt) mit den Belastungskomponenten

$$
p_{r}=-\gamma t \cos \varphi, \quad p_{\varphi}=\gamma t \sin \varphi,
$$

so erhält man die erstmals von Milankovič̃ angegebene Kuppel gleicher Festigkeit. Der Mittelflächenmeridian genügt der Differentialgleichung

$$
\frac{d \varphi}{d r}=\frac{\gamma}{\sigma_{0}}-\frac{1}{r} \operatorname{tg} \varphi
$$

deren Lösung sich nicht in geschlossener Form darstellen läßt. Verwendet man als Parameter auf dem Meridian ausnahmsweise die Zylinderkoordinate $z$, so erhält man für den Verlauf der Schalenstärke $t=t(z)$ die Beziehung

$$
t=t_{0} e^{\frac{\gamma}{\sigma_{0}} z}
$$

Für den hydrostatischen Innendruck

$$
p_{r}=\gamma z, \quad p_{\varphi}=0
$$

( $\gamma=$ spezifisches Gewicht der Füllung) ergibt die Bedingung (2.1) den sogenannten Tropfenbehälter ${ }^{3}$ mit der Meridianbedingung

$$
\frac{1}{r_{1}}+\frac{1}{r_{2}}=\frac{\gamma}{\sigma_{0} t_{0}} z
$$

Durch die Bedingung (2.1) ist die Festigkeit des Materials offenbar optimal ausgenützt, wenn für das Fließen (bzw. den Bruch) die größte Normalspannung maßgebend ist, doch anderseits sind auch die Form der Schale und die Schalenstärke durch die vorgegebene Belastung vollständig be-

1 W. Flügge, Statik und Dynamik der Schalen, 2. Aufl., S. 37, Berlin, Göttingen, Heidelberg 1957.

2 M. Milankovič, Arbeiten der Jugoslaw. Akad. d. Wissensch. Agram 175 (1908) S. 140. Vergleiche auch W. Flügge, a. a. O. und besonders K. Federhofer, Bauing. 20 (1939) S. 366.

3 W. Flügge, a. a. O., S. 39. 
stimmt. Die Beschränkung scheint wenig sinnvoll, wenn für das Fließen (bzw. den Bruch) nicht die größte Normalspannung verantwortlich ist, sondern das Material einer anderen Fließbedingung (bzw. Bruchhypothese) genügt, so zum Beispiel derjenigen von Tresca bzw. derjenigen von $v$. Mises.

H. Ziegler ${ }^{1}$ hat daher einen alten Ansatz von C. B. Biezeno ${ }^{2}$ wieder aufgegriffen und den Begriff der gleichen Festigkeit in einer neuen Form verwendet. Er ersetzt die stark einschränkende Forderung (2.1) durch die Fließbedingung von Tresca (bzw. Bruchhypothese konstanter Schubspannung)

$$
\operatorname{Max}\left(\left|\sigma_{\varphi}\right|,\left|\sigma_{\vartheta}\right|,\left|\sigma_{\varphi}-\sigma_{\vartheta}\right|\right)=\sigma_{0},
$$

oder durch die Fließbedingung von v. Mises (bzw. die Bruchhypothese konstanter Gestaltänderungsarbeit)

$$
\sigma_{\varphi}^{2}+\sigma_{\vartheta}^{2}-\sigma_{\varphi} \sigma_{\vartheta}=\sigma_{0}^{2} .
$$

In der Spannungsebene $\left(\sigma_{\varphi}, \sigma_{\vartheta}\right)$ (Abb. 3) wird die Bedingung (2.1) durch den Punkt $A$ (bzw. $D$ ) die Bedingung (2.5) durch das Sechseck von Tresca und (2.6) durch die umgeschriebene Ellipse dargestellt.

Schalen, die der Bedingung (2.5) genügen und daher von gleicher Festigkeit im Sinne der Bedingung von Tresca sind, nennen wir fortan $T$-Schalen. Entsprechend wollen wir Schalen, die die Bedingung (2.6) erfüllen, als $M$-Schalen bezeichnen.

Anstatt durch zwei Gleichungen, wie bei der Bedingung (2.1), sind durch (2.5) oder (2.6) die Spannungen $\sigma_{\varphi}$ und $\sigma_{\vartheta}$ nur noch durch eine einzige Beziehung miteinander verknüpft. Man hat also sozusagen einen Freiheitsgrad mehr, und es ist eine interessante Frage, ob sich eine vorgegebene Mittelfläche zu einer Schale gleicher Festigkeit im Sinne der Bedingung (2.5) bzw. (2.6) ausbilden läßt. Wie wir später sehen werden, hängt ihre Beantwortung wesentlich von

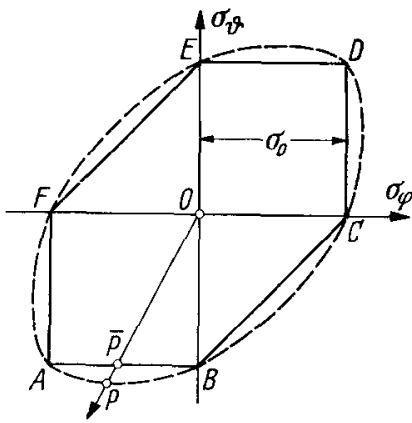

Abb. 3. Fließfiguren (Bruchfiguren) in der Spannungsebene $\left(\sigma_{\varphi}, a_{\eta}\right)$. der vorgegebenen Belastung ab.

Aus Symmetriegründen sind im Scheitel einer Rotationsschale die beiden Schnittkräfte gleich groß, und es gilt daher die Gleichung

$$
N_{\varphi}(0)=N_{\vartheta}(0) \text {. }
$$

Ist nun (2.5) bzw. (2.6) vorgeschrieben und damit die Festigkeit des Materials vollkommen ausgenützt, so muß für den Scheitel der Schale der Spannungspunkt $P$ in der Spannungsebene $\left(\sigma_{\varphi}, \sigma_{\vartheta}\right)$ (Abb. 3) mit $A$ (bzw. D) zusammenfallen, und damit muß

$$
\sigma_{\varphi}(0)=\sigma_{\vartheta}(0)=\mp \sigma_{0}
$$

sein. Trifft man noch die selbstverständliche Annahme, daß die Schalenstärke im Scheitel größer als null ist, so folgt aus (1.14), (1.16), (1.9) und (2.7), daß das obere Vorzeichen von (2.8) nur für $p_{r}(0)<0$ und das untere nur für $p_{r}(0)>0$ gilt.

Damit sich also eine gegebene Mittelfläche zu einer Schale gleicher Festigkeit [nach (2.1), oder nach (2.5) bzw. (2.6)] ausbilden läßt, ist die Bedingung

$$
p_{r}(0) \neq 0
$$

notwendig. Diese Bedingung ist aber beinahe immer erfüllt. Der einzige Ausnahmefall tritt auf, wenn sich die verschiedenen Anteile von $p_{r}(0)$ (Eigengewicht usw.) gerade aufheben.

3. Schalen mit den Lastkomponenten $p_{\varphi}=p_{\varphi}(\varphi)$ und $p_{r}=p_{r}(\varphi)$. Wir betrachten in diesem Abschnitt eine fest gewählte Mittelfläche, die den Voraussetzungen des Abschnitts 1 genügt, mit der Meridiangleichung $r=r(p)$. Nach (1.8) ist für $\varphi$ höchstens ein Intervall

$$
0 \leq \varphi \leq \varphi^{*}<\pi
$$

zulässig, wobei $\varphi^{*}$ den Definitionsbereich von $r(\varphi)$ nach oben begrenzt. Die beiden Winkel $\varphi$ und $\vartheta$ (wie im Abschnitt 1 definiert) verwenden wir als Flächenparameter.

Für eine rotationssymmetrische Last können die Lastkomponenten $p_{\varphi}$ und $p_{r}(\mathrm{Abb} .2)$ noch von $\varphi$ und der Schalenstärke $t$ (z. B. Eigengewicht) abhängen. Wir setzen in diesem Abschnitt

1 H. Ziegler, Ing.-Arch. 26 (1958) S. 378.

2 C. B. Biezeno, De Ingenieur 39 (1922) S. 781. 
speziell voraus, daß die Lasten $p_{\varphi}$ und $p_{r}$ nur stetige Funktionen von $\varphi$ allein sind, und daß $p_{r}$ noch der Bedingung (2.9) genüge. Damit haben wir Lasten vernachlässigt oder zum voraus durch Funktionen von $\varphi$ approximiert, die auch von der Schalenstärke $t$ abhängen.

Wir zeigen in diesem Abschnitt, daß man für die gegebene Mittelfläche und für gegebene Lasten der obigen Art die Schalenstärke auf genau eine Art so wählen kann, daß eine T-Schale bzw. $M$ Schale entsteht. Dabei werden wir von den spezielleu Eigenschaften der Funktioneu $r(\varphi), p_{\varphi}(\varphi)$ und $p_{r}(\varphi)$ keinen Gebrauch machen. Die folgenden U̇berlegungen gelten daher für beliebige Schalen und Lasten, die den einleitend formulierten Voraussetzungen genügen.

Mit Hilfe der Beziehungen

und

$$
\frac{N_{\varphi}}{r_{1}}+\frac{N_{\vartheta}}{r_{2}}=p_{r}(\varphi)
$$

$$
N_{\varphi}=\frac{1}{r_{2} \sin ^{2} \varphi}\left[\int r_{1} r_{2}\left(p_{r} \cos \varphi-p_{\varphi} \sin \varphi\right) \sin \varphi d \varphi+c\right]
$$

ist die Berechnung der Schnittkräfte auf eine Quadratur zurückgeführt, da der Integrand von (3.2) eine stetige Funktion von $\varphi$ allein ist. Man kann auch die Formel (3.2) direkt erhalten, indem man das Schalenstück zwischen dem Scheitel und einem Breitenkreis betrachtet, und für dieses die Gleichgewichtsbedingung in Richtung der Rotationsachse formuliert. Mit (1.7) erhält man dann

$$
N_{\varphi}=\frac{1}{r_{2} \sin ^{2} \varphi} \int_{0}^{\varphi} r_{1} r_{2}\left(p_{r} \cos \psi-p_{\varphi} \sin \psi\right) \sin \psi \mathrm{d} \psi
$$

wobei sich die Bestimmung der Integrationskonstanten erübrigt, da man zwischen festen Grenzen integriert. Eine einfache Diskussion der Beziehung (3.3) zeigt, daß die Schnittkraft $N_{\varphi}$ für alle Werte von $\varphi$, für die der Integrand definiert ist, stetig verläuft. Nach (3.1), (1.6) und (1.7) gilt das Gleiche auch für die Schnittkraft $N_{\vartheta}$. Für $q=0$ crhält man aus (3.1), (1.9) und (2.7) die Gleichung

$$
N_{\varphi}(0)=N_{\vartheta}(0)=\frac{r_{1}(0) p_{r}(0)}{2} .
$$

a) Die Schalenstärke $t=t(\varphi)$ für eine $M$-Schale. Für eine gegebene Mittelfläche mit einer gegebenen Belastung lassen sich die Schnittkräfte $N_{\varphi}$ und $N_{\vartheta}$ mit den Formeln (3.1) und (3.3) als Funktionen von $\varphi$ berechnen. Wir kennen daher die Schalenstärke $t$ als Funktion von $\varphi$, wenn es uns gelingt, die Schalenstärke als Funktion der Größen $N_{\varphi}, N_{\vartheta}$ darzustellen.

Nach (2.6) müssen die Spannungen $\sigma_{\varphi}$ und $\sigma_{\vartheta}$ einer $M$-Schale der Bedingung

genügen. Mit den Gleichungen (1.16)

$$
\sigma_{\varphi}^{2}+\sigma_{\vartheta}^{2}-\sigma_{\varphi} \sigma_{\vartheta}=\sigma_{0}^{2}
$$

$$
\sigma_{\varphi}=\frac{N_{\varphi}}{t}, \quad \sigma_{\vartheta}=\frac{N_{\vartheta}}{t}
$$

ergibt sich für die Schalenstärke $t$, wenn man aus den beiden letzten Beziehungen die Spannungen eliminiert, die Formel

$$
t=\frac{1}{\sigma_{0}} \sqrt{N_{\varphi}^{2}+N_{\vartheta}^{2}-N_{\varphi} N_{\vartheta}}
$$

wobei nur das positive Vorzeichen der Wurzel sinnvoll ist. Da der Radikand positiv definit ist, gilt die Formel (3.7) für alle Werte $\varphi$, für welche die Schnittkräfte definiert sind, falls nicht gerade beide Schnittkräfte gleichzeitig verschwinden. Für den Scheitel ist das wegen (3.4), (1.9) und (2.7) offensichtlich nicht möglich. Dank der Stetigkeit der Schnittkräfte ist nun die Rotationsfläche zumindest in einer Umgebung des Scheitels auf genau eine Art als $M$-Schale ausbildbar. Es gilt der

Satz 1. Eine gegebene Rotationsfläche mit der Meridiangleichung $r=r(\varphi)\left(0 \leq \varphi \leq \varphi^{*}<\pi\right)$, die den drei Voraussetzungen des ersten Abschnitts und deren Belastung den Annahmen dieses Abschnitts genügt, läßt sich im Intervall $0 \leq \varphi \leq \varphi^{* *} \leq \varphi^{*}$ auf genau eine Art als $M$-Schale ausbilden. Der Winkel $\varphi^{* *}$ ist entweder gleich $\varphi^{*}$ oder durch das erstmalige gleichzeitige Verschwinden der beiden Schnittkräfte $N_{\varphi}$ und $N_{\vartheta}$ bestimmt.

Die Lösungen der obigen Art genügen zwar allen Gleichungen, doch sind sie nicht immer bis zum Winkel $\varphi^{*}$ zulässig. Es kann nämlich der Fall eintreten, daß die Schalenstärke $t$ sehr stark anwächst. Für dicke Schalen gilt aber die Membrantheorie nicht mehr. Leider läßt sich diese Grenze nicht allgemein durch eine Formel darstellen, weil sie wesentlich von der speziellen Wahl der Funktionen $r(\varphi), p_{r}(\varphi)$ und $p_{\varphi}(\varphi)$ abhängt. 